\title{
Supporting Information for \\ Oxygen Isotope Exchange over Copper-containing Mordenite: the Effect of Copper Loading and Si/Al Ratio
}

Mikalai A. Artsiusheuskia, , Jeroen A. van Bokhoven ${ }^{a, b}$, Vitaly L. Sushkevich ${ }^{\mathrm{b}, *}$

${ }^{a}$ Laboratory for Catalysis and Sustainable Chemistry, Paul Scherrer Institut, 5232 Villigen PSI, Switzerland

${ }^{b}$ Institute for Chemistry and Bioengineering, ETH Zurich, Vladimir-Prelog-Weg 1, 8093 Zurich, Switzerland

*Corresponding author: Tel.: +41563103518; E-mail address: vitaly.sushkevich@psi.ch, 

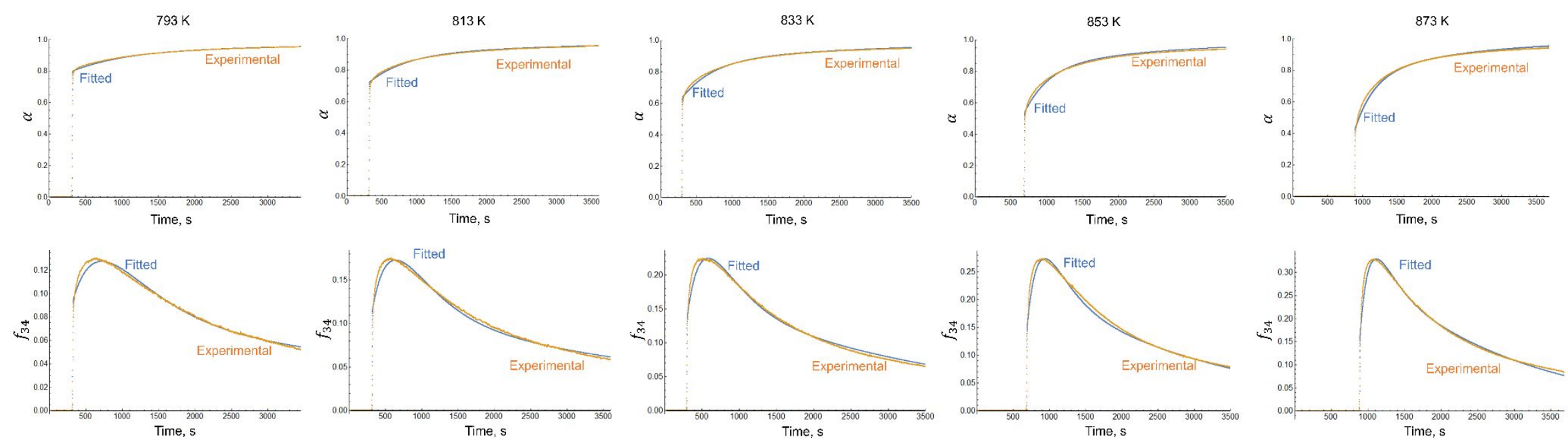

Figure S1. Experimental data points (orange) and the fitted curves (blue) for parameters $f_{34}$ and $\alpha$ for the isothermal isotope exchange experiment over $\mathrm{Cu}(4.3) \mathrm{MOR}(6)$ 

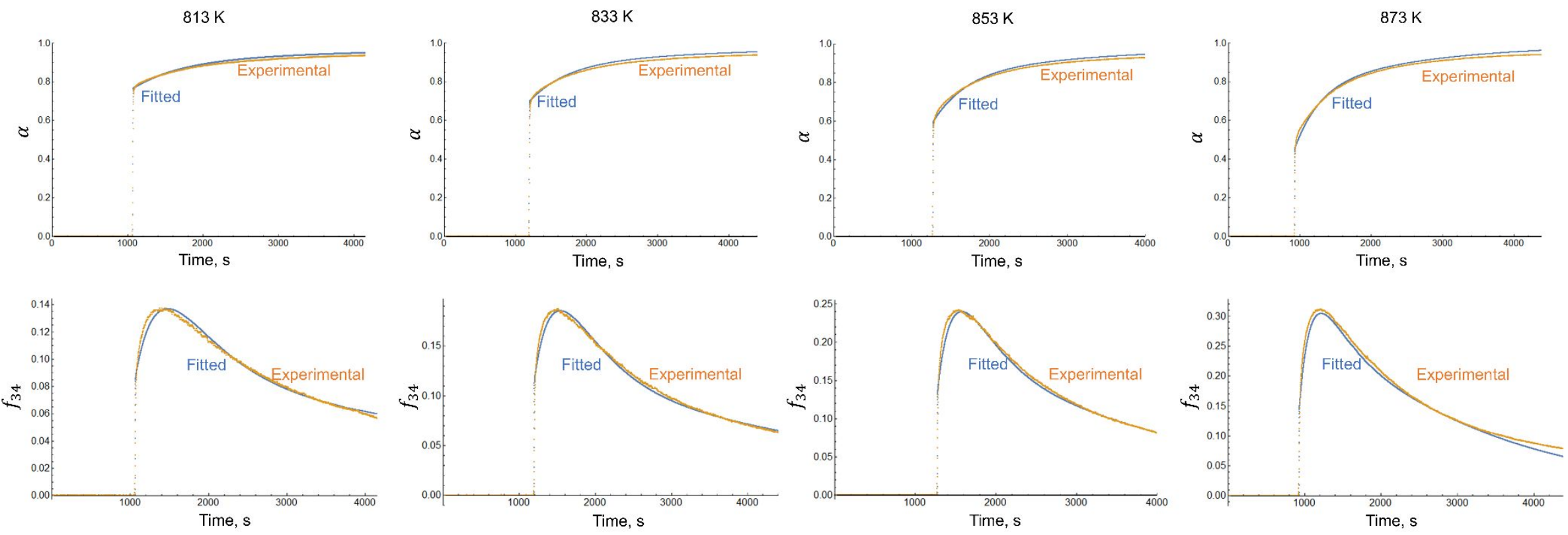

Figure S2. Experimental data points (orange) and the fitted curves (blue) for parameters $f_{34}$ and $\alpha$ for the isothermal isotope exchange experiment over Cu(3.5)MOR(6) 
$813 \mathrm{~K}$
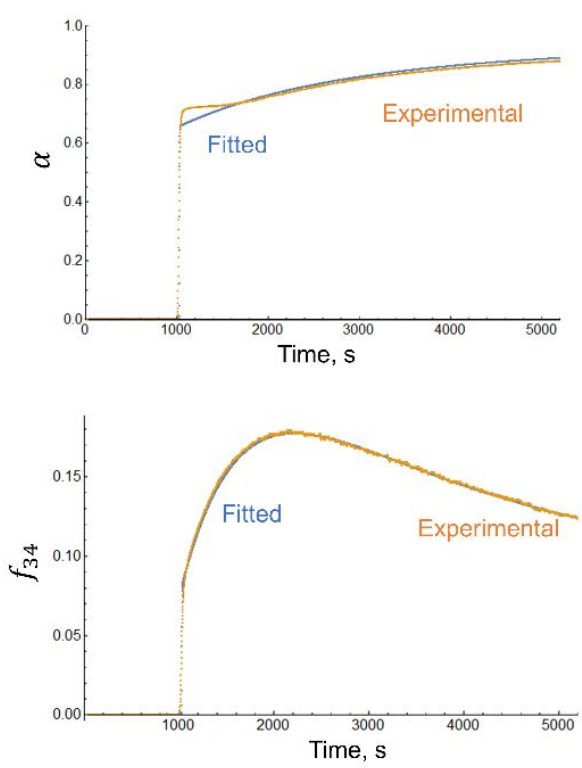

$833 \mathrm{~K}$
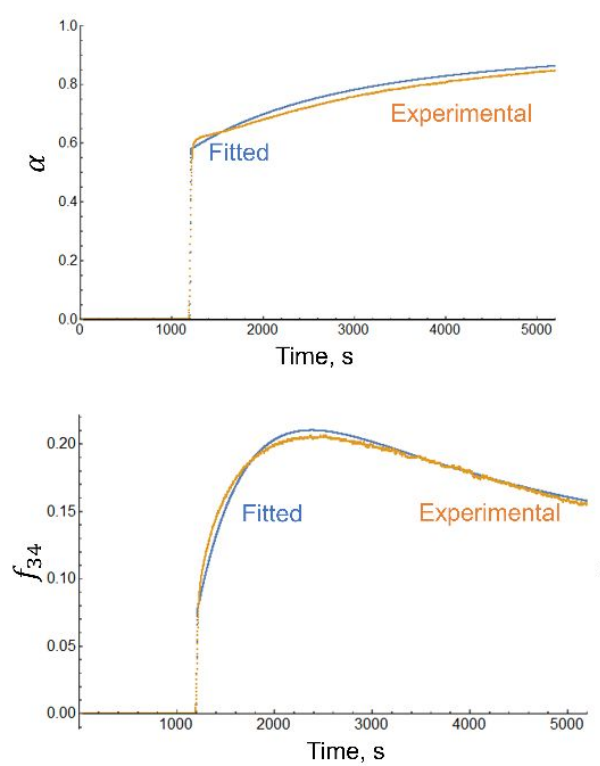

$853 \mathrm{~K}$
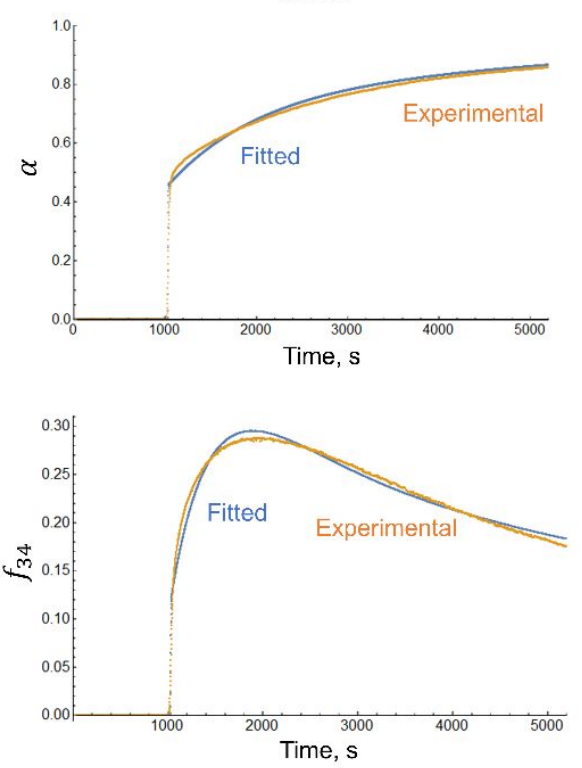

$873 \mathrm{~K}$
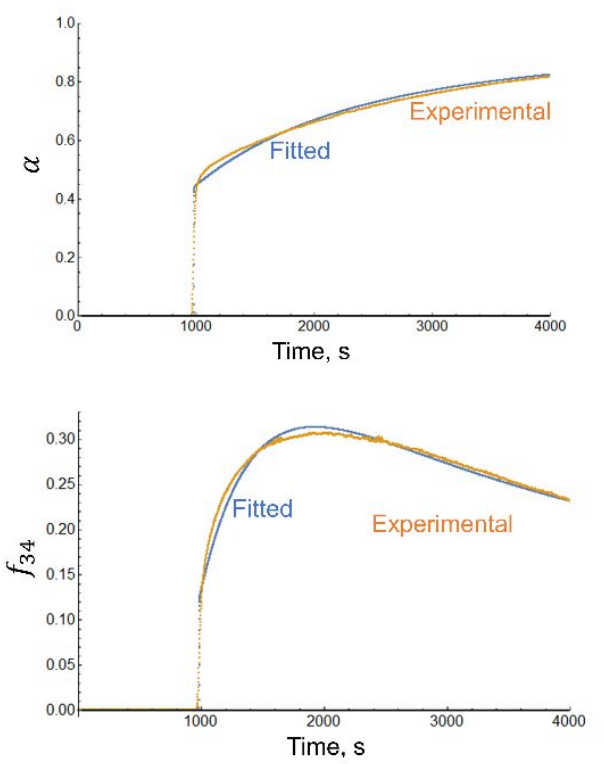

Figure S3. Experimental data points (orange) and the fitted curves (blue) for parameters $f_{34}$ and $\alpha$ for the isothermal isotope exchange experiment over Cu(2.5)MOR(6) 
$833 \mathrm{~K}$
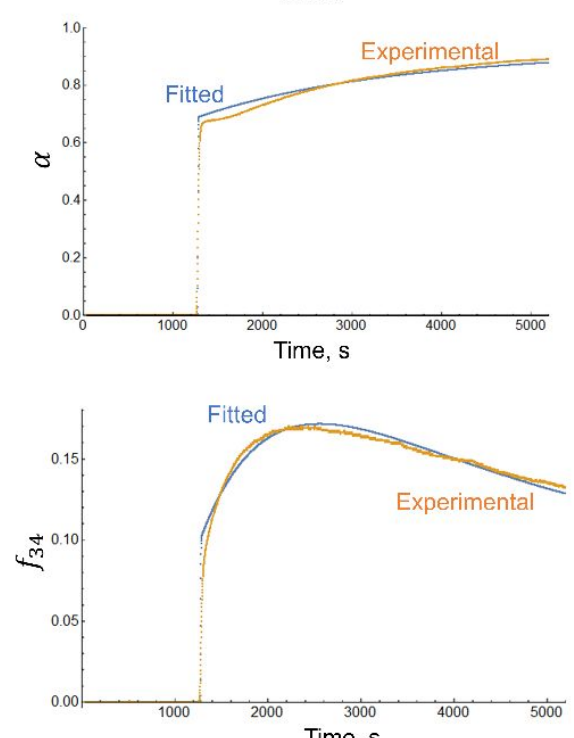

$853 \mathrm{~K}$
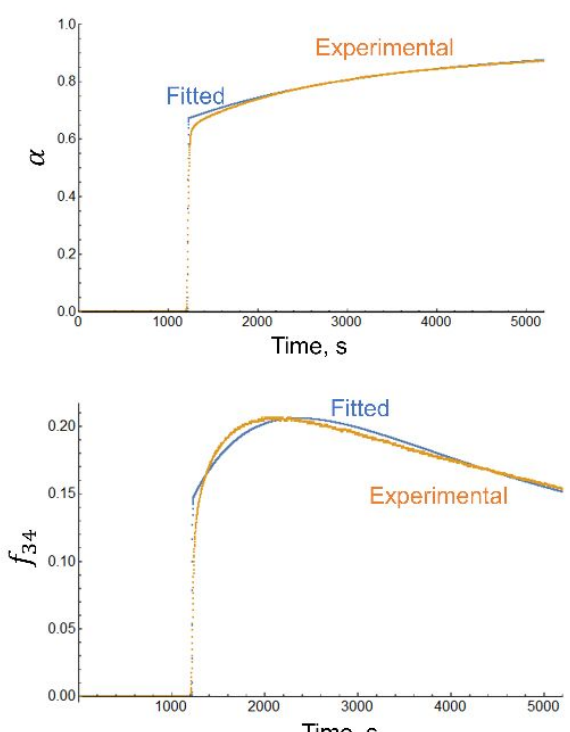

$873 \mathrm{~K}$
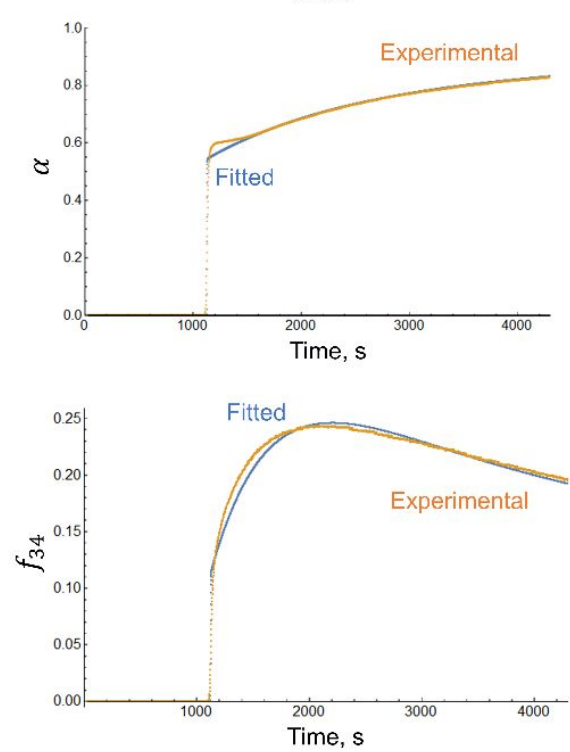

$893 \mathrm{~K}$
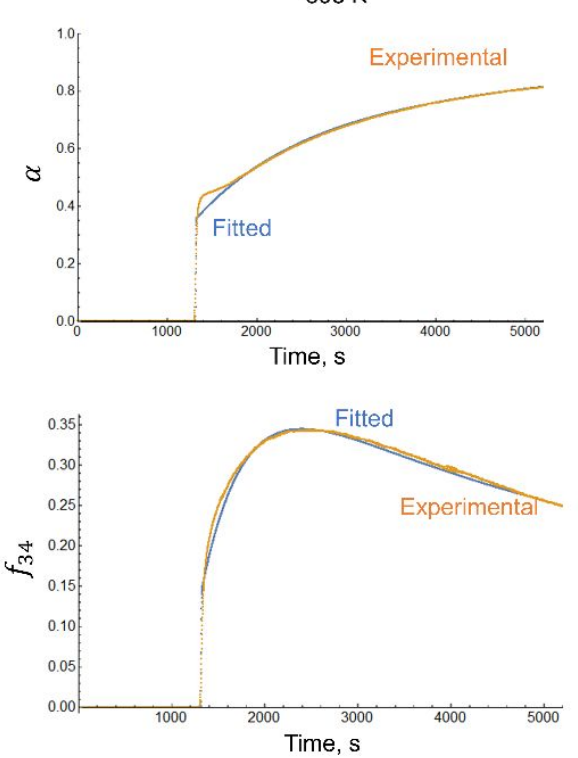

Figure S4. Experimental data points (orange) and the fitted curves (blue) for parameters $f_{34}$ and $\alpha$ for the isothermal isotope exchange experiment over Cu(1.7)MOR(6) 
873 K
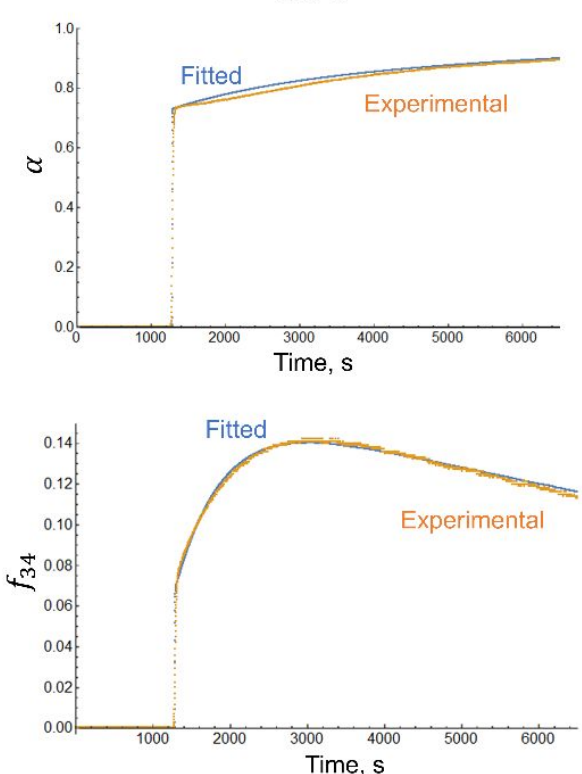

$893 \mathrm{~K}$
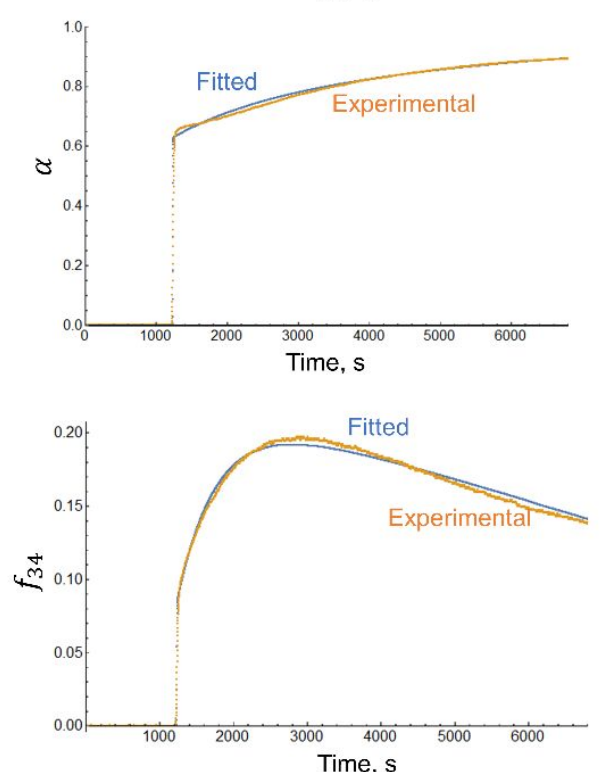

$913 \mathrm{~K}$
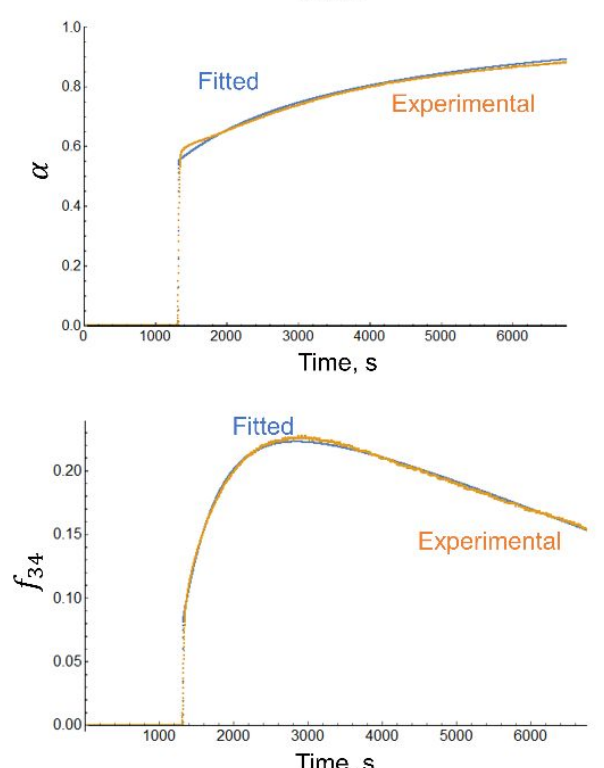

$933 \mathrm{~K}$
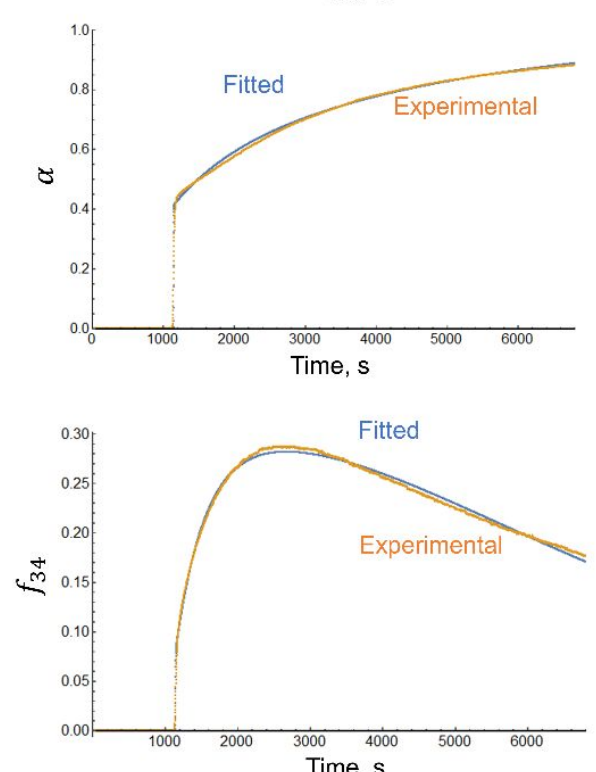

Figure S5. Experimental data points (orange) and the fitted curves (blue) for parameters $f_{34}$ and $\alpha$ for the isothermal isotope exchange experiment over Cu(3.4)MOR(10) 

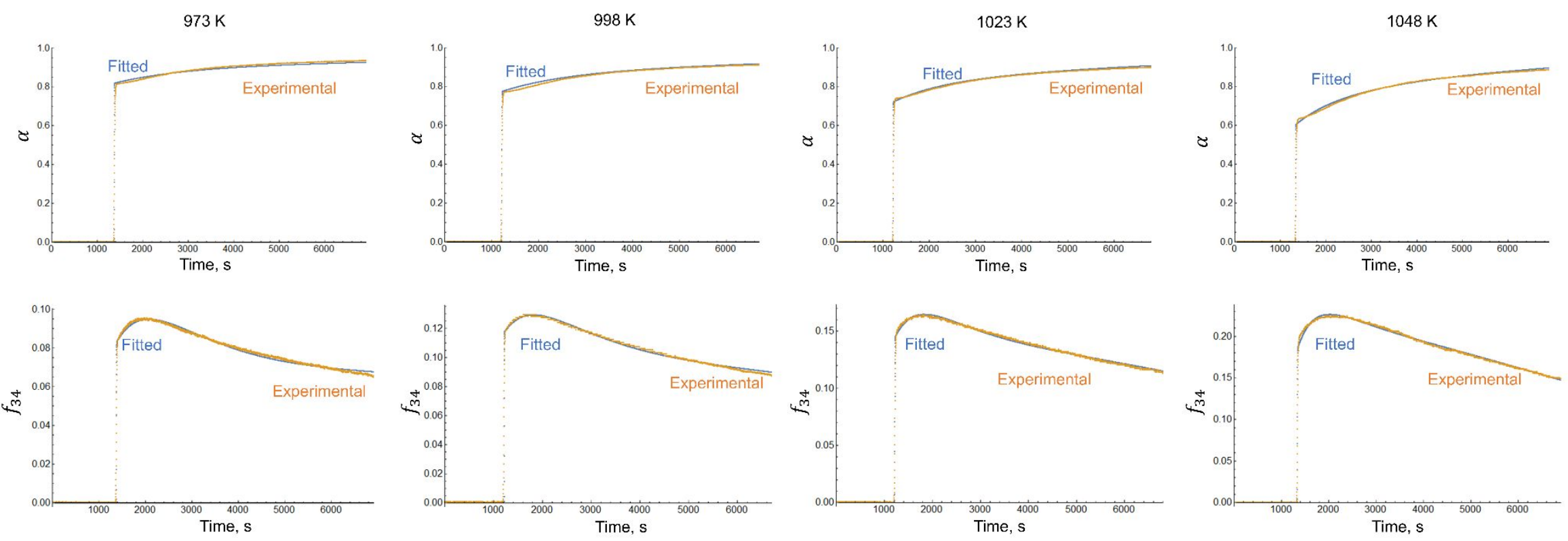

Figure S6. Experimental data points (orange) and the fitted curves (blue) for parameters $f_{34}$ and $\alpha$ for the isothermal isotope exchange experiment over Cu(1.2)MOR(46) 


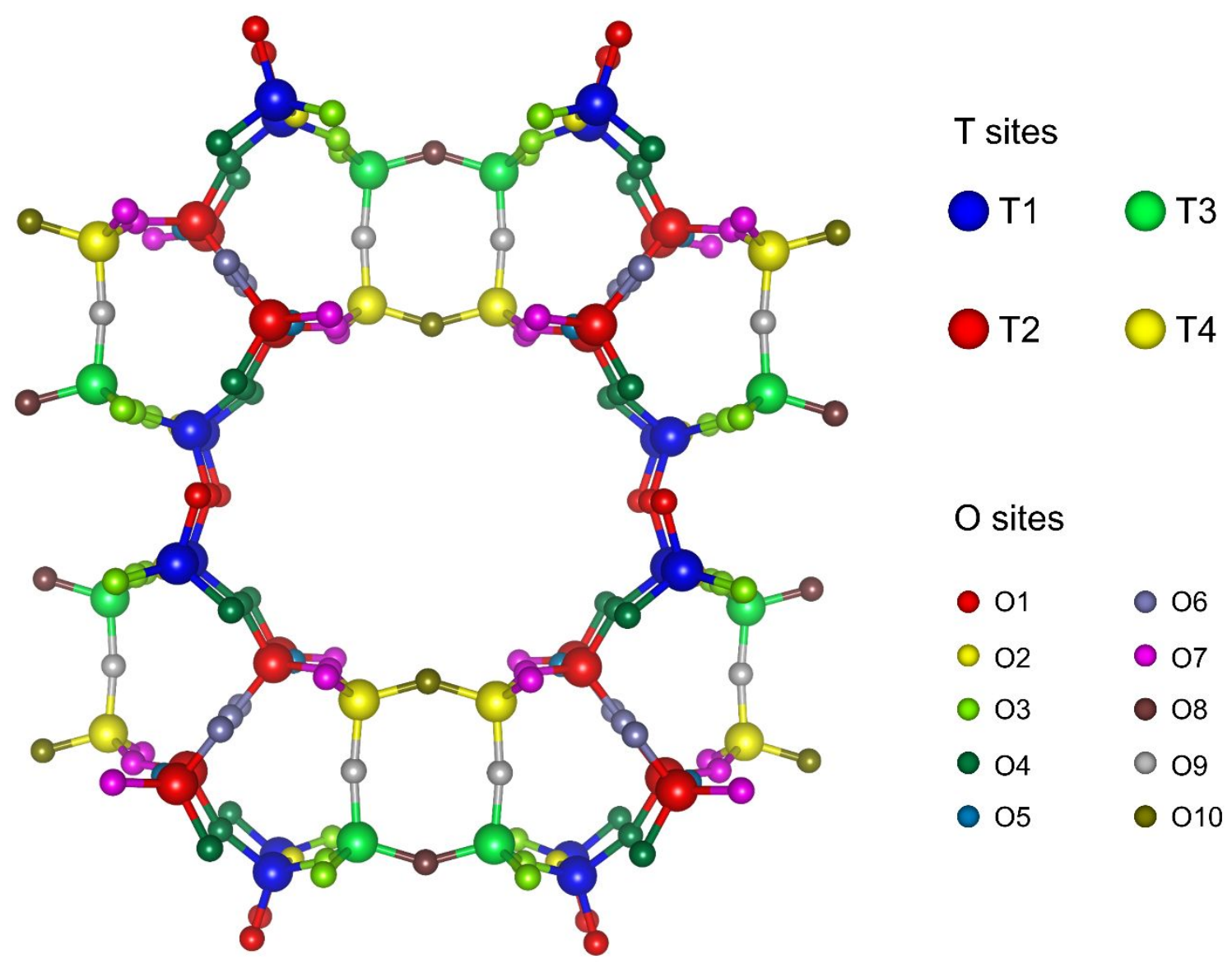

Figure S7. The different crystallographic positions for $\mathrm{Si}$ or $\mathrm{Al}$ atoms (T sites) and for oxygen atoms (O sites). 